\title{
Clinical outcomes and safety of apatinib monotherapy in the treatment of patients with advanced epithelial ovarian carcinoma who progressed after standard regimens and the analysis of the $V E G F R 2$ polymorphism
}

\author{
ZHEN YAN $^{1 *}$, YUAN-YUAN GU ${ }^{2 *}$, XIAO-DI HU ${ }^{1}$, QUN ZHAO ${ }^{1}$, \\ HAI-LI KANG ${ }^{1}$, MIAO WANG ${ }^{1}$, WEI DUAN ${ }^{1}$ and YIN GUAN ${ }^{2}$ \\ ${ }^{1}$ Department of Gynecological Oncology, Beijing Obstetrics and Gynecology Hospital, \\ Capital Medical University, Beijing 100026; ${ }^{2}$ Department of Oncology, Beijing Chao-Yang Hospital, \\ Capital Medical University, Beijing 100020, P.R. China
}

Received December 9, 2019; Accepted June 5, 2020

DOI: $10.3892 / \mathrm{ol} .2020 .11857$

\begin{abstract}
The aims of the present study were to investigate the clinical outcomes and safety of apatinib monotherapy in the treatment of patients with advanced epithelial ovarian carcinoma (EOC) who have progressed after standard regimens, and to analyze the vascular endothelial growth factor receptor 2 (VEGFR2) rs2071559 polymorphism. A total of 118 patients with advanced EOC who received apatinib treatment were included in the study. Tumor response was evaluated using progression-free survival (PFS) and overall survival (OS) time, and safety data were documented. Additionally, peripheral blood and peripheral blood mononuclear cell (PBMC) specimens from the patients with EOC were collected to perform the genotyping of genetic polymorphism and assess the mRNA expression of $V E G F R 2$, respectively. The objective response rate across the 118 patients with advanced EOC was $38.98 \%$, the disease control rate was $63.56 \%$, the median PFS time was 4.65 months and the median OS time was 15.10 months. Regarding the polymorphism analysis, the prevalence of rs2071559 in VEGFR2 among the 118 patients
\end{abstract}

Correspondence to: Professor Wei Duan, Department of Gynecological Oncology, Beijing Obstetrics and Gynecology Hospital, Capital Medical University, 251 Pan Jiayuan Road, Beijing 100026, P.R. China

E-mail: wduan@163.net

Professor Yin Guan, Department of Oncology, Beijing Chao-Yang Hospital, Capital Medical University, 8 Gongti South Road, Beijing 100020, P.R. China

E-mail: guanyin_cyyy@163.com

*Contributed equally

Key words: ovarian carcinoma, apatinib, VEGFR2, polymorphism, clinical outcomes, safety with advanced EOC was recorded as the TT genotype in 72 cases $(61.02 \%)$, TC genotype in 41 cases $(34.75 \%)$ and CC genotype in 5 cases $(4.23 \%)$, and the minor allele frequency of rs2071559 was 0.22 . The distribution of the three genotypes was in accordance with the Hardy-Weinberg equilibrium $(\mathrm{P}=0.781)$. TC and CC genotypes were merged in the subsequent analysis. The prognosis analyses suggested that the median PFS time of patients with the TC/CC genotype and the TT genotype was 3.10 and 5.40 months, respectively $(\mathrm{P}=0.015)$. Moreover, the median OS time of the two genotypes was 12.60 and 17.50 months, respectively $(\mathrm{P}=0.009)$. However, no association was noted between genotype status of the polymorphism and adverse reactions. Additionally, the mRNA expression analysis indicated that the mRNA expression levels of VEGFR2 in PBMC specimens were significantly different between TT and TC/CC genotypes $(\mathrm{P}<0.001)$. The present study suggested that the clinical outcomes of patients with advanced EOC, who progressed after standard regimens and received apatinib treatment, might be influenced by the VEGFR2 rs2071559 polymorphism.

\section{Introduction}

Ovarian carcinoma is a common gynecological malignancy and one of the leading causes of cancer-associated death in women worldwide. In 2018 alone, it accounted for 295,400 new cases and $\sim 184,800$ deaths globally (1). Annually, there are $\sim 52,100$ new cases and $\sim 22,500$ associated deaths in China (2). The majority patients with ovarian carcinoma are diagnosed with epithelial ovarian carcinoma (EOC), which accounts for $\sim 90 \%$ of ovarian carcinoma cases (3). Most of the patients are diagnosed with advanced disease due to the non-specific symptoms (4). Breakthroughs in treatment over the past two decades have improved the prognosis of EOC slightly, but the 5-year overall survival rate is still $<30 \%$ for patients with advanced-stage EOC (5). According to European Society for Medical Oncology and National Comprehensive Cancer Network (NCCN) guidelines for ovarian 
carcinoma $(6,7)$, the standard initial chemotherapeutic treatment for patients with stage II-IV ovarian carcinoma is based on carboplatin plus paclitaxel regimens. Those who relapse $\geq 6$ months or $<6$ months after initial chemotherapy are termed as platinum-sensitive patients and platinum-resistant patients, respectively (8). Almost all patients with recurrent disease eventually develop platinum resistance (9). The available second-line treatment regimens are liposomal doxorubicin, and weekly paclitaxel, topotecan, gemcitabine and etoposide, respectively, as single agents. These second-line treatment regimens have demonstrated an objective response rate (ORR) ranging from $10-30 \%$ and a median progression-free survival (PFS) time of 3-4 months $(10,11)$. No appropriate regimens were available for subsequent lines of treatment (12).

It has been demonstrated that angiogenesis plays an important role in tumor growth, recurrence and metastasis of EOC (13). Anti-angiogenic therapy consists of anti-vascular endothelial growth factor (VEGF) antibodies and small molecule anti-angiogenesis tyrosine kinase inhibitors (TKIs) (14). Bevacizumab, a monoclonal antibody, has been approved for the therapy of ovarian cancer (15). Although a previous study showed that the combination of bevacizumab with chemotherapy in ovarian cancer improves the ORR, the administration of bevacizumab is ineffective and the prognosis is clinically unsatisfactory (11). Pazopanib was the first anti-angiogenic TKI, with positive clinical significance in a phase II clinical trial, to be recommended by NCCN guidelines of ovarian cancer (16). Subsequently, sorafenib demonstrated promising clinical outcomes as a maintenance therapy for patients with platinum-resistant ovarian cancer (17). These studies suggest the potential therapeutic significance of anti-angiogenic TKIs in the treatment of EOC.

Apatinib was the first anti-angiogenic TKI with demonstrable efficacy and safety in patients with advanced gastric cancer (18). Additionally, a previous study supported the effectiveness of apatinib in EOC (19). Generally, the clinical application of anti-angiogenic drugs results in a relatively low ORR. In advanced EOC, the ORR of monotherapy with pazopanib, sorafenib and apatinib were recorded as 18 (16), 3.4 (20) and $41.4 \%$ (21), respectively. Therefore, these individual differences between TKIs show that the clinical application of apatinib has a promising effect in patients with EOC. Unfortunately, no biomarker for patients with EOC who received apatinib treatment is available to determine prognosis and monitor disease progression clinically.

An important therapeutic target of apatinib is VEGF receptor 2 (VEGFR2), which is located on chromosome $4 \mathrm{q} 12$ and contains 30 exons (22). To date, there are few studies investigating the polymorphism of VEGFR2 in the Chinese population. rs2071559 is located in the upstream region of VEGFR2 (23). A previous study indicated that rs2071559 is significantly associated with a pathological complete response in patients with advanced breast cancer treated with capecitabine-based neoadjuvant therapy (24). Furthermore, studies involving European and American populations have suggested that the VEGFR2 polymorphism is associated with improved prognosis in patients with advanced renal cell carcinoma who are treated with sorafenib. However, the underlying mechanisms for this effect has not been completely interpreted or understood (25).
Therefore, the present study aimed to explore the clinical outcomes and safety of apatinib monotherapy in the treatment of patients with advanced EOC who progressed after standard regimens, and to analyze the VEGFR2 rs2071559 polymorphism.

\section{Materials and methods}

Study design and therapeutic schedule. The present study was designed as a retrospective analysis considering patients with ovarian carcinoma receiving apatinib treatment. Therefore, patients with advanced ovarian carcinoma that progressed after standard regimens between January 2015 and December 2018 in the Department of Gynecological Oncology of Beijing Obstetrics and Gynecology Hospital (Beijing, China) were enrolled in this study. The eligibility criteria included: i) Histologically or cytologically confirmed diagnosis of epithelial ovarian carcinoma confirmed by a pathological expert; ii) pathological stage III or IV according to the International Federation of Gynecology and Obstetrics (FIGO) staging system (26); iii) female sex and age $\geq 18$ years; iv) Eastern Cooperative Oncology Group (ECOG) performance status of $0-2$; v) patients treated with apatinib after at least two failed standard lines of treatment, including patients that had undergone secondary cytoreductive surgery after relapse; and vi) at least one measurable target lesion according to Response Evaluation Criteria in Solid Tumors (RECIST 1.1) (27). The exclusion criteria included: i) Presence of concomitant tumors or serious diseases; ii) hemoptysis $>50 \mathrm{ml}$ per day; iii) presence of uncontrolled hypertension or serious presence of protein in the urine; and iv) a diagnosis of squamous cell skin cancer or in situ cancer of the cervix uteri. A flow chart of the study is illustrated in Fig. 1. The primary endpoint of the study was PFS time, the secondary endpoint was ORR, overall survival (OS) time and the analysis of VEGFR2 gene polymorphism.

Apatinib was administered at the initial dosage of 500 or $750 \mathrm{mg}$ per day, orally with warm water, $30 \mathrm{~min}$ after meals, and for 28 days as one cycle, until disease progression or intolerable adverse reactions. The precise dose of apatinib was determined according to the baseline physical conditions of the patients, namely body surface area, ECOG score and age. The dose of apatinib was adjusted according to the hematological (neutropenia) or non-hematological toxicity (hypertension) during the treatment. The treatment was discontinued when a potentially life-threatening adverse reaction occurred. The present study was approved by the Ethics Committee of Beijing Obstetrics and Gynecology Hospital (Beijing, China). Written informed consent was provided by enrolled patients or by their relatives.

The clinical outcomes were assessed according to RECIST 1.1 criteria (27). The change of target lesions was assessed with computed tomography (CT) scans after the completion of the first cycle and then after every two cycles, or more frequently if the clinical symptoms of the patients deteriorated. The occurrence of adverse reactions during treatment was evaluated using the Common Terminology Criteria for Adverse Events version 4.03, in order to register hematological and non-hematological events that may be drug-related (28). Adverse events classified as grade $\geq 2$ with an incidence $\geq 10 \%$ were recorded and analyzed. 


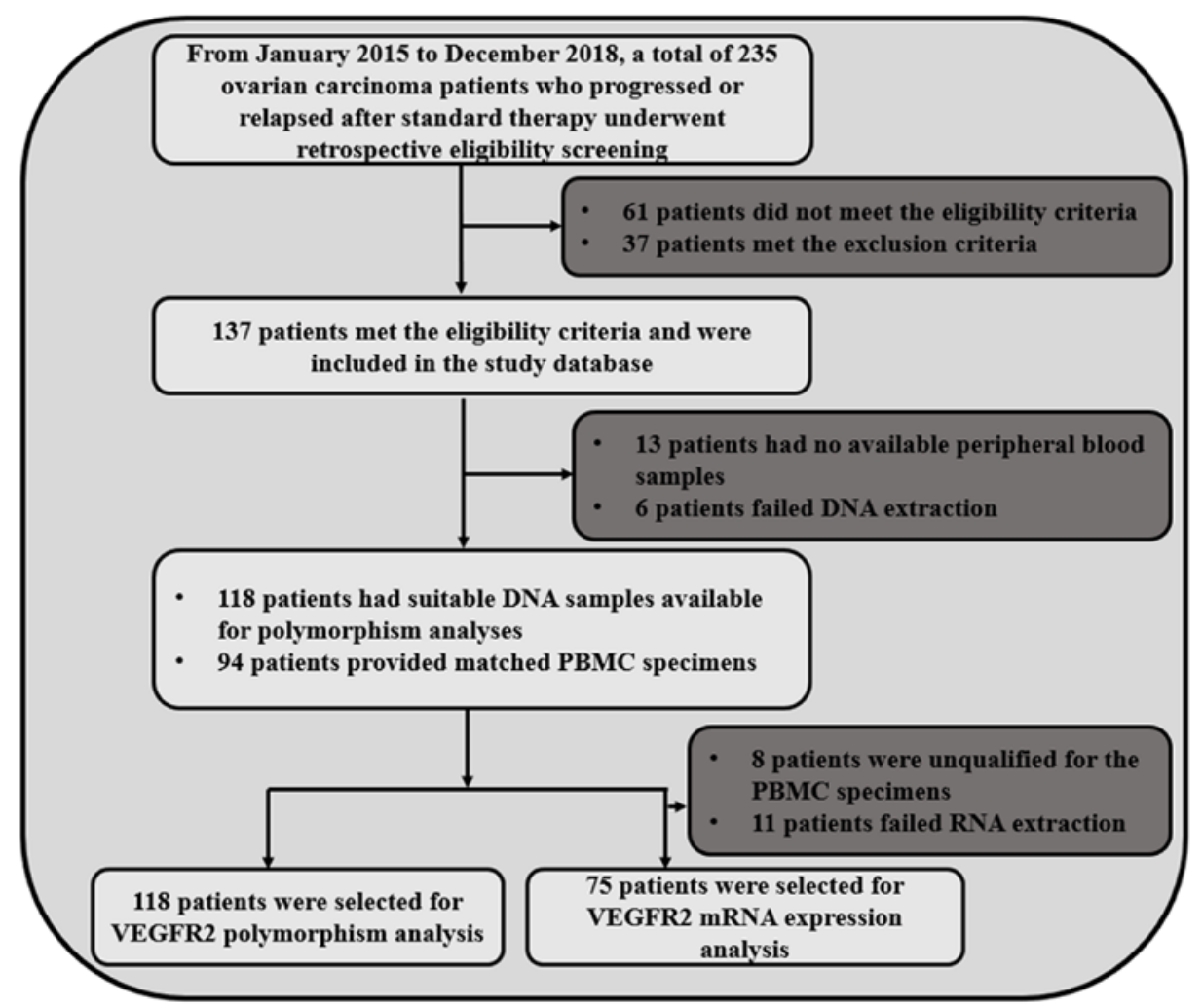

Figure 1. Flow chart of the retrospective study of apatinib monotherapy in the treatment of patients with advanced epithelial ovarian carcinoma who progressed after standard regimens. PBMC, peripheral blood mononuclear cells; VEGFR2, vascular endothelial growth factor receptor 2.

Collection of peripheral blood specimens and genotyping of VEGFR2 polymorphism. Genomic DNA was extracted from whole venous blood collected prior to treatment with apatinib during hospitalization using phenol chloroform methods, according to the standard clinical procedure. Single nucleotide polymorphisms of $V E G F R 2$, with the minor allele frequency $>10 \%$ in the Chinese population, were identified using the National Center for Biotechnology Information database (https://www.ncbi.nlm.nih.gov/snp/), and included rs2071559, rs2305948 and rs11941492. As shown in Table I, of the three polymorphisms analyzed in the 118 patients with EOC, only rs2071559 was significantly associated with PFS time. Therefore, the subsequent analysis of this study was focused on the rs2071559 polymorphism. The rs2071559 polymorphism of $V E G F R 2$ was genotyped through polymerase chain reaction-restriction fragment length polymorphism (PCR-RFLP) (Sangon Biotech Co., Ltd.). Initially, the PCR product including this polymorphism was amplified, using SYBRGreen (Sangon Biotech Co., Ltd.). The forward primer was 5'-TCACTAGGGCTCTTCGTTGG-3' and the reverse primer was 5'-GAAGCGGATACTCAGCCAAG-3'. PCR primers were synthesized by Sangon Biotech Co., Ltd. PCR reaction conditions were as follows: $94^{\circ} \mathrm{C}$ for $5 \mathrm{~min}$; 36 cycles at $94^{\circ} \mathrm{C}$ for $45 \mathrm{sec}, 63.5^{\circ} \mathrm{C}$ for $45 \mathrm{sec}$ and $72^{\circ} \mathrm{C}$ for $30 \mathrm{sec} ; 72^{\circ} \mathrm{C}$ for $10 \mathrm{~min}$. In total, $2 \mu 1$ PCR products (size, $271 \mathrm{bp})$ were digested using the restriction enzyme Bsm I (Thermo Fisher Scientific Inc.). Agarose gel electrophoresis (1\%) was performed on the enzyme digestion products. The genotypes of the polymorphism were determined by the size of PCR bands as follows: TT genotype (one 271-bp band); CC genotype (one 108-bp band and one 163-bp band); and
TC genotype (one 271-bp band, one 108-bp band and one 163-bp band).

Collection of peripheral blood mononuclear cell (PBMC) specimens and analysis of VEGFR2 mRNA expression. Initially, the PBMC specimens were collected from 94 randomly matched samples in the 118 patients with EOC. However, 8 PBMC specimens were not available and RNA extraction failed in 11 PBMC specimens. Eventually, a total of 75 PBMC specimens were available for further analysis and preserved in liquid nitrogen. Total RNA samples were extracted using the TRIzol ${ }^{\circledR}$ reagent (Bao Biological Engineering Co. Ltd.) according to the manufacturer's instructions and stored at $-80^{\circ} \mathrm{C}$ for mRNA expression analysis. In total, $500 \mathrm{ng}$ RNA extracted from each PBMC specimen was used as a template for reverse transcription-PCR to prepare the first strand of cDNA using the PrimeScript RT reagent kit (Bao Biological Engineering Co., Ltd.) according to the manufacturer's instructions. Relative quantitative analysis of VEGFR2 mRNA expression was performed on the Roche LightCycler ${ }^{\circledR}$ 480 using a SYBR Premix EX Taq system (Bao Biological Engineering Co., Ltd.). The forward primer of VEGFR2 was 5'-ATGCAGAGCAAGGTGCTGC-3' and the reverse primer was 5'-TTAAACAGGAGGAGAGCTCAGTG-3'. The amplification reaction $(20 \mu \mathrm{l})$ contained $10 \mu \mathrm{l}$ SYBR Premix EX Taq, $0.2 \mu 1$ each primer $(20 \mu \mathrm{M}), 7.6 \mu 1$ double distilled water (ddH2O) and $2 \mu \mathrm{l}$ cDNA. PCR reaction conditions were as follows: $95^{\circ} \mathrm{C}$ for $5 \mathrm{~min} ; 40$ cycles at $95^{\circ} \mathrm{C}$ for $10 \mathrm{sec} ; 62^{\circ} \mathrm{C}$ for $10 \mathrm{sec}$; and $72^{\circ} \mathrm{C}$ for $10 \mathrm{sec}$. The mRNA expression of VEGFR2 was detected using a comparative Cq method $\left(2^{-\Delta \Delta C q}\right)(29)$. The mRNA expression of GAPDH was used as an endogenous 
Table I. Details of the polymorphisms included in the present study and the preliminary analysis between genotypes status and PFS.

\begin{tabular}{|c|c|c|c|c|c|}
\hline Polymorphism & Primers (5'-3') & Location & MAF & Median PFS time, months & P-value \\
\hline rs2071559 & $\begin{array}{l}\text { F: TCACTAGGGCTCTTCGTTGG } \\
\text { R: GAAGCGGATACTCAGCCAAG }\end{array}$ & Upstream region & 0.22 & 5.4 vs. 3.1 (TT vs. TC/CC) & 0.015 \\
\hline rs2305948 & $\begin{array}{l}\text { F: TTCCAAGACCATAGCTTACCA } \\
\text { R: AATGTTTACCAAAGCCCAGA }\end{array}$ & Coding region & 0.15 & 4.7 vs. 4.3 (CC vs. CT/TT) & 0.315 \\
\hline rs11941492 & $\begin{array}{l}\text { F: TTGAGTTCCAATCTCAGCTTCA } \\
\text { R: CTGGCCTTGAGAAAATCACC }\end{array}$ & Intron region & 0.28 & 4.5 vs. 4.8 (CC vs. CT/TT) & 0.553 \\
\hline
\end{tabular}

MAF, minor allele frequency; PFS, progression-free survival; F, forward; R, reverse.

control. The forward primer of GAPDH was 5'-GCACCGTCA AGGCTGAGAAC-3' and the reverse primer was 5'-TGGTGA AGACGCCAGTGGA-3'.

Statistical analysis. All variables in this study were statistically analyzed using the statistical analysis software SPSS version 25.0 (IBM Corp.). Hardy-Weinberg equilibrium was measured for the rs2071559 genotypes using the $\chi^{2}$ test. The significance of observed differences in proportions was assessed using the $\chi^{2}$ test, and Fisher's exact test was performed when the dataset was small. The analysis between continuous variables and genotype status was performed using the Mann-Whitney $\mathrm{U}$ non-parametric test (between two groups). The primary endpoint was PFS. The Kaplan-Meier curves were drawn using Stata 14.0 (StataCorp LP) to compare the differences in PFS and OS times according to each genotype status. Survival differences were compared using a log-rank test. PFS was defined as the period from the initial treatment with apatinib up until disease progression or patient death, whichever occurred first. OS was defined as the period from the time of treatment with apatinib to patient death from any cause. For those without disease progression or death by the end of the study follow-up, the survival end points were censored at the date of last follow-up. For the multivariable analysis, a Cox proportional hazards model was constructed for PFS time, and the backward selection procedure was used to adjust for potential confounding covariates. $\mathrm{P}<0.05$ was considered to indicate a statistically significant difference.

\section{Results}

Baseline characteristics of the 118 patients with EOC and genotypes of VEGFR2 rs2071559 polymorphism. The baseline characteristics of the 118 patients with EOC are shown in Table II. The median age of the patients was 57 years (range, 41-78 years) and the ECOG 0 score was reported in 55 patients. According to FIGO stage criteria, stage III was noted in 92 patients. The most common histology of EOC was serous, diagnosed in 87 patients. Regarding the first-line platinum-based chemotherapy response, 34 patients were identified with platinum-refractory disease, 49 patients with platinum-resistant disease and 35 patients were platinum-sensitive. In terms of the tumor differentiation, the majority of patients (58 in total) were found to present with poorly differentiated tumors. Two lines of previous treatment were reported in 21 patients and $\geq 3$ lines of previous treatment were confirmed in 97 patients. Apatinib was administered at an initial dosage of 500 and $750 \mathrm{mg}$ in 86 and 32 patients, respectively. However, a dosage reduction had to be implemented in a total of 48 patients due to hematological or non-hematological toxicities.

Of the VEGFR2 polymorphisms analyzed, only rs2071559 was of clinical significance (Table I). The germline mutation frequency of rs2071559 among the 118 patients with EOC were as follows: TT genotype, 72 cases (61.02\%); TC genotype, 41 cases (34.75\%); CC genotype, 5 cases (4.23\%), and the minor allele frequency of rs 2071559 was 0.22 . The distribution of the three genotypes was in accordance with the Hardy-Weinberg equilibrium ( $\mathrm{P}=0.781)$. TC and $\mathrm{CC}$ genotypes were merged in the subsequent analysis. As shown in Table II, patients with TT and TC/CC genotypes were well balanced, presenting similar baseline characteristics. No significant differences were observed between the two groups.

Influence of VEGFR2 rs2071559 polymorphism on the clinical outcomes of the 118 patients with EOC. All 118 patients with EOC included in this study were subjected to efficacy evaluation during the treatment with apatinib, where the best overall response of each patient was recorded. A complete response was not observed in any of the patients, whereas a partial response was identified in 46 patients, stable disease in 29 patients and progression disease in 43 patients. According to RECIST 1.1, the ORR was $38.98 \%$ and disease control rate (DCR) was $63.56 \%$. The waterfall plot representing the best percentage change in the dimensions of the target lesion is shown in Fig. 2.

The last follow-up time of this study was June 2019. The median follow-up time of all patients from the time of enrollment to the last follow-up was 14.50 months (range, 1-36 months). The median PFS time of the 118 patients with EOC included in this study was 4.65 months [ $95 \%$ confidence interval (CI), 3.45-5.80]. Additionally, the median PFS time according to different baseline characteristic was further analyzed (Fig. 3). Univariate analysis according to baseline characteristics suggested that ECOG score $(\mathrm{P}=0.015)$, FIGO stage $(\mathrm{P}=0.008)$ and tumor differentiation $(\mathrm{P}=0.019)$ were significantly associated with PFS time. In addition, the results indicated that the median PFS time of patients with an ECOG score of 0 was significantly longer compared with that of 
Table II. Baseline characteristics of the 118 patients with EOC according to TT $(n=72)$ or TC/CC ( $=46)$ vascular endothelial growth factor receptor 2 rs2071559 polymorphism status.

4397T>C genotypes

\begin{tabular}{|c|c|c|c|c|}
\hline & & & & \\
\hline Characteristics & Value & TT & $\mathrm{TC} / \mathrm{CC}$ & P-value \\
\hline Median age (range), years & $57(41-81)$ & $57(41-79)$ & $58(44-81)$ & 0.632 \\
\hline ECOG score, n (\%) & & & & 0.832 \\
\hline 0 & $55(46.61)$ & $33(45.83)$ & $22(47.83)$ & \\
\hline $1-2$ & $63(53.39)$ & $39(54.17)$ & $24(52.17)$ & \\
\hline FIGO stage, $\mathrm{n}(\%)$ & & & & 0.951 \\
\hline III & 92 (77.97) & $56(77.78)$ & $36(78.26)$ & \\
\hline IV & $26(22.03)$ & $16(22.22)$ & $10(21.74)$ & \\
\hline Histology of EOC, n (\%) & & & & 0.971 \\
\hline Serous & $87(73.73)$ & $53(73.61)$ & $34(73.91)$ & \\
\hline Mixed & $31(26.27)$ & 19 (26.39) & $12(26.09)$ & \\
\hline First-line platinum response, $\mathrm{n}(\%)$ & & & & 0.793 \\
\hline Platinum-refractory & $34(28.81)$ & $20(27.78)$ & $14(30.43)$ & \\
\hline Platinum-resistant, $<6$ months & $49(41.53)$ & $29(40.28)$ & $20(43.48)$ & \\
\hline Platinum-sensitive, $\geq 6$ months & $35(29.66)$ & $23(31.94)$ & $12(26.09)$ & \\
\hline Tumor differentiation, $\mathrm{n}(\%)$ & & & & 0.949 \\
\hline Well differentiated & $19(16.10)$ & $11(15.28)$ & $8(17.39)$ & \\
\hline Intermediately differentiated & $41(34.75)$ & $25(34.72)$ & $16(34.78)$ & \\
\hline Poorly differentiated & $58(49.15)$ & $36(50.00)$ & $22(47.83)$ & \\
\hline Lines of previous treatment regimens, $\mathrm{n}(\%)$ & & & & 0.927 \\
\hline 2 & $21(17.80)$ & $13(18.06)$ & $8(17.39)$ & \\
\hline$\geq 3$ & $97(82.20)$ & $59(81.94)$ & $38(82.61)$ & \\
\hline Initial dosage of apatinib in $\mathrm{mg}, \mathrm{n}(\%)$ & & & & 0.517 \\
\hline 500 & $86(72.88)$ & $54(75.00)$ & $32(69.57)$ & \\
\hline 750 & $32(27.12)$ & $18(25.00)$ & $14(30.43)$ & \\
\hline Dose of reduction, $\mathrm{n}(\%)$ & & & & 0.154 \\
\hline Yes & $48(40.68)$ & $33(45.83)$ & $15(32.61)$ & \\
\hline No & $70(59.32)$ & $39(54.17)$ & $31(67.39)$ & \\
\hline
\end{tabular}

ECOG, Eastern Cooperative Oncology Group; EOC, epithelial ovarian carcinoma; FIGO, International Federation of Gynecology and Obstetrics.

patients with a score of 1-2 (5.55 vs. 3.90 months), the median PFS time of patients with FIGO stage III was significantly higher compared with that of patients with stage IV (5.40 vs. 3.25 months) and the median PFS time of patients with well and intermediate differentiation was significantly superior compared with that of patients with poor differentiation (5.25 and 4.70 vs. 3.95 months).

The analysis of the effect of the VEGFR2 rs2071559 polymorphism in terms of the PFS, showed that the median PFS time between patients with TT and TC/CC genotypes was significantly different, being 5.40 and 3.10 months, respectively $\left(\chi^{2}=6.50\right)$ (Fig. 4). Furthermore, a Cox regression model was constructed. Results of the multivariate analysis are shown in Table III. After adjustment for other confounding factors, a significant difference was observed for the influence of TC/CC genotype of rs2071559 on PFS time. Results indicated that the rs2071559 polymorphism was an independent factor for PFS time [hazard ratio (HR), 1.69; $\mathrm{P}=0.021$ ]. Additionally, ECOG high score $(\mathrm{HR}, 1.41 ; \mathrm{P}=0.018)$, FIGO IV stage $(\mathrm{HR}, 1.81$; $\mathrm{P}=0.011)$ and tumor poor differentiation $(\mathrm{HR}, 1.28 ; \mathrm{P}=0.046)$ were also independent factors for PFS.

The evaluation of OS in the present study showed that the median OS time of the 118 patients with EOC was 15.10 months (95\% CI, 13.10-17.25). Regarding the effect of the VEGFR2 rs2071559 polymorphism on OS, the results showed that the median OS time between patients with TT and TC/CC genotypes was significantly different, with an OS of time 12.60 and 17.50 months, respectively $\left(\chi^{2}=4.50\right)($ Fig. 5).

Association between VEGFR2 rs2071559 polymorphism and the safety of patients with EOC. Adverse reactions classified as grade $\geq 2$ with an incidence $\geq 10 \%$ were analyzed. The most common treatment-related adverse reactions were hand-foot syndrome, hypertension, nausea/vomiting, proteinuria, fatigue and diarrhea, with an incidence of 40.68, 25.42, $18.64,13.56,11.86$ and $10.17 \%$, respectively (Table IV). The 


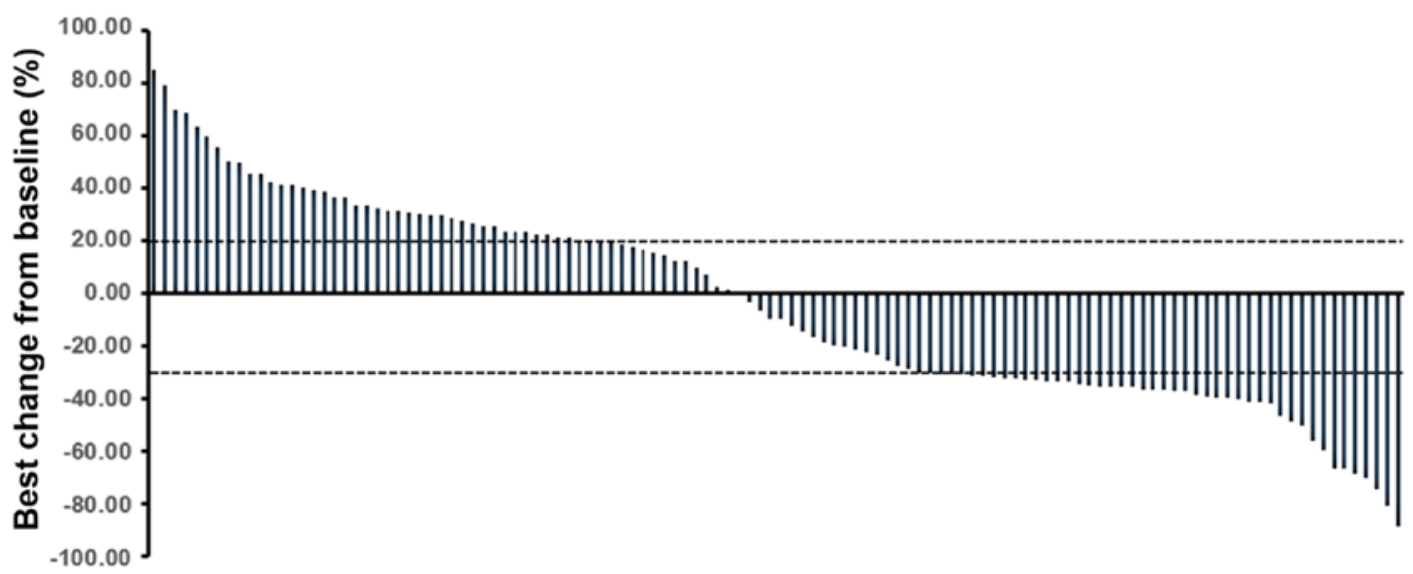

Figure 2. Waterfall plot of best change (reductions in sum of lesion diameters) from baseline (before the start of apatinib treatment) in the 118 patients with epithelial ovarian carcinoma receiving apatinib monotherapy.

\begin{tabular}{|c|c|c|c|c|c|c|}
\hline \multirow[t]{2}{*}{ Characteristics } & \multirow[t]{2}{*}{$\mathbf{N}$} & & \multirow[t]{2}{*}{ mPFS (Months) } & \multicolumn{2}{|c|}{$95 \% \mathrm{Cl}$} & \multirow[t]{2}{*}{ P-value } \\
\hline & & & & Lower & Upper & \\
\hline Total & 118 & $\longmapsto$ & 4.65 & 3.45 & 5.80 & \\
\hline Age (years) & & & & & & 0.531 \\
\hline$>57$ & 54 & $\longmapsto 1$ & 4.55 & 3.30 & 5.65 & \\
\hline$\leq 57$ & 64 & $\mapsto$ & 4.73 & 3.51 & 5.83 & \\
\hline ECOG & & & & & & 0.015 \\
\hline 0 & 55 & $\mapsto-1$ & 5.55 & 4.85 & 6.20 & \\
\hline $1-2$ & 63 & $\mapsto r$ & 3.90 & 3.10 & 4.95 & \\
\hline FIGO stage & & & & & & 0.008 \\
\hline III & 92 & $\because$ & 5.40 & 4.65 & 6.15 & \\
\hline IV & 26 & $\mapsto-1$ & 3.25 & 2.65 & 3.75 & \\
\hline Histology of EOC & & & & & & 0.611 \\
\hline Serous & 87 & $\longrightarrow$ & 4.78 & 3.68 & 5.70 & \\
\hline Mixed & 31 & . & 4.45 & 3.55 & 5.30 & \\
\hline First line platinum response & & & & & & 0.323 \\
\hline Platinum refractory & 34 & $\vdash$ & 4.41 & 3.52 & 5.40 & \\
\hline Platinum resistant ( $<6$ months) & 49 & $\longrightarrow$ & 4.70 & 3.25 & 6.10 & \\
\hline Platinum sensitive ( $\geqslant 6$ months) & 35 & $\longrightarrow$ & 4.75 & 3.50 & 5.89 & \\
\hline Tumor differentiation & & & & & & 0.019 \\
\hline Well differentiated & 19 & $\cdot=$ & 5.25 & 4.10 & 6.05 & \\
\hline Intermediate differentiated & 41 & $\mapsto$ & 4.70 & 3.50 & 5.75 & \\
\hline Poorly differentiated & 58 & 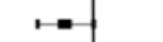 & 3.95 & 3.20 & 4.75 & \\
\hline Lines of previous treatment regimer & & & & & & 0.655 \\
\hline 2 & 21 & $\mapsto$ & 4.80 & 3.55 & 6.05 & \\
\hline$\geqslant 3$ & 97 & $1-1$ & 4.50 & 3.50 & 5.85 & \\
\hline Initial dosage of apatinib & & & & & & 0.532 \\
\hline $500 \mathrm{mg}$ & 86 & $\longmapsto$ & 4.65 & 3.30 & 5.75 & \\
\hline $750 \mathrm{mg}$ & 32 & $\mapsto$ & 4.65 & 3.35 & 5.90 & \\
\hline
\end{tabular}

Figure 3. Forest plot of mPFS of the 118 patients with EOC score data according to different baseline characteristics subgroups. CI, confidence interval; ECOG, Eastern Cooperative Oncology Group; EOC, epithelial ovarian carcinoma; FIGO, International Federation of Gynecology and Obstetrics; mPFS, median progression-free survival; N, number of patients.

evaluation of the effect of the rs2071559 polymorphism on the incidence of adverse events grade $\geq 2$ showed no significant difference between the patients with the TT and TC/CC genotypes.

Association between rs2071559 polymorphism and the $m R N A$ expression of VEGFR2. Across the 75 specimens included, the prevalence of the VEGFR2 rs2071559 polymorphism was as follows: TT genotype, 46 patients $(61.33 \%)$; TC genotype, 26 patients (34.67\%); and CC genotype, 3 cases (4.00\%). The prevalence was comparable with the genotype frequency observed among the 118 patients with EOC. The distribution of the three genotypes was in accordance with the Hardy-Weinberg equilibrium $(\mathrm{P}=0.776)$. Similarly, $\mathrm{CC}$ and TC genotypes were merged in the subsequent analysis, and patients with the TC/CC genotype showed a significantly 
Table III. Multivariate Cox regression analysis of progression-free survival according to baseline characteristics and vascular endothelial growth factor receptor 2 rs2071559 polymorphism.

\begin{tabular}{lccr}
\hline Characteristics & HR $(95 \% \mathrm{CI})$ & $\mathrm{df}$ & P-value \\
\hline ECOG score & & 1 & 0.018 \\
0 & $1.00^{\mathrm{a}}$ & & \\
$1-2$ & & & 0.011 \\
FIGO stage & $1.00^{\mathrm{a}}$ & 1 & 0.046 \\
III & $1.81(1.38-2.36)$ & 1 & 0.021 \\
IV & $1.00^{\mathrm{a}}$ & & \\
Tumor differentiation & $1.28(1.02-1.51)$ & 1 & \\
Well and intermediately differentiated & & & \\
Poorly differentiated & $1.00^{\mathrm{a}}$ & & \\
rs2071559 genotype & $1.69(1.21-1.95)$ & & \\
TT genotype & & & \\
TC/CC genotype &
\end{tabular}

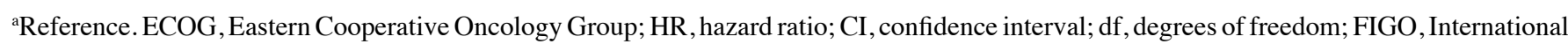
Federation of Gynecology and Obstetrics.

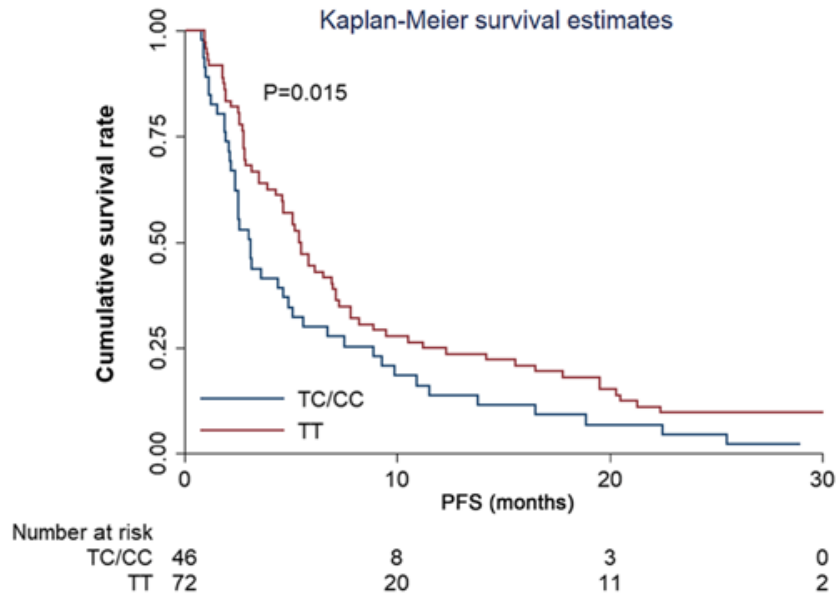

Figure 4. PFS of the 118 patients with epithelial ovarian carcinoma according to the vascular endothelial growth factor receptor 2 rs2071559 genotype status. PFS, progression-free survival.

higher relative expression of VEGFR2 mRNA in PBMC specimens compared with those with the TT genotype $(4.06 \pm 0.331$ vs. $2.90 \pm 0.480$; Fig. 6).

\section{Discussion}

The present retrospective study provides evidence regarding the clinical outcomes and safety of apatinib in the treatment for patients with EOC who progressed after standard regimens. Polymorphism analysis indicated that patients with the TC/CC genotype of the rs 2071559 polymorphism were associated with worse PFS and OS times. Furthermore, the results suggested that the mRNA expression levels of VEGFR2 were significantly different according to rs2071559 genotype status. Therefore, the clinical outcomes of patients with EOC receiving apatinib therapy may be influenced by the VEGFR2

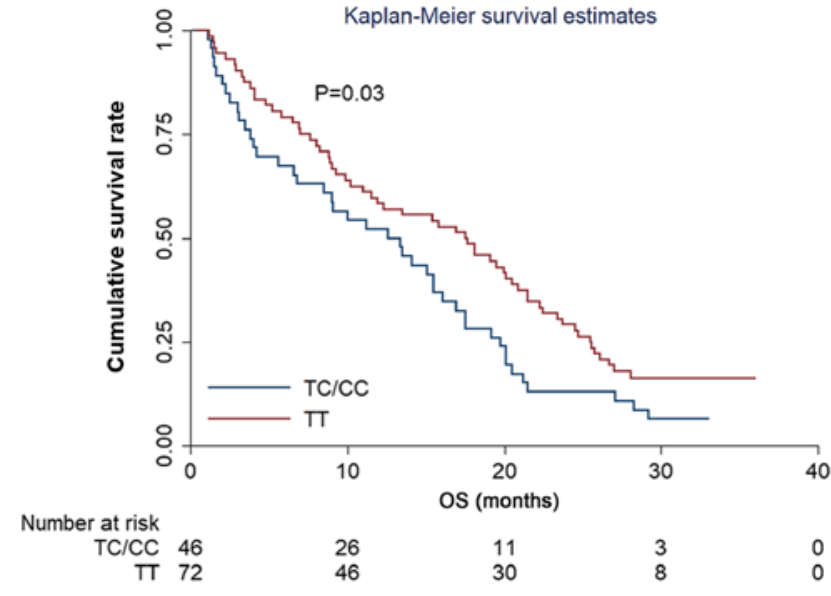

Figure 5. OS of the 118 patients with EOC according to the vascular endothelial growth factor receptor 2 rs 2071559 genotype status. OS, overall survival.

rs2071559 polymorphism through the mediation of VEGFR2 mRNA expression.

As an anti-angiogenic TKI, apatinib has been demonstrated to be effective in gastric cancer, colorectal cancer, lung cancer, soft tissue sarcoma and gynecological tumors (30-33). In the present study, the clinical outcomes of 118 patients with EOC receiving apatinib treatment were evaluated. The ORR was $38.98 \%$, the DCR was $63.56 \%$ and the median PFS time was 4.65 months, all values that were lower than those reported in the phase II clinical trial of apatinib in recurrent EOC by Miao et al (21), where ORR was $41.4 \%$, DCR was $68.9 \%$ and median PFS time was 5.10 months. It was hypothesized that the reason for these discrepancies maybe attributed to the retrospective design of the present study, with insufficient patient management compared with clinical trials, which was also observed in the other retrospective study, and the clinical outcomes in this retrospective study were inferior to that in 
Table IV. Analysis between vascular endothelial growth factor receptor 2 rs2071559 genotype status and adverse reactions classified as grade $\geq 2$.

rs2071559 genotype status, $\mathrm{n}(\%)$

\begin{tabular}{lcrrr}
\cline { 3 - 4 } Adverse reactions & Total, $\mathrm{n}(\%)$ & $\mathrm{TT}(\mathrm{n}=72)$ & $\mathrm{TC} / \mathrm{CC}(\mathrm{n}=46)$ & P-value \\
\hline Hand-foot syndrome & $48(40.68)$ & $30(41.67)$ & $18(39.13)$ & 0.784 \\
Hypertension & $30(25.42)$ & $19(26.39)$ & $11(23.91)$ & 0.763 \\
Nausea/vomiting & $22(18.64)$ & $14(19.44)$ & $8(17.39)$ & 0.780 \\
Proteinuria & $16(13.56)$ & $10(13.89)$ & $6(13.04)$ & 0.896 \\
Fatigue & $14(11.86)$ & $9(12.50)$ & $5(10.87)$ & 0.789 \\
Diarrhea & $12(10.17)$ & $8(11.11)$ & $4(8.70)$ & 0.672 \\
\hline
\end{tabular}

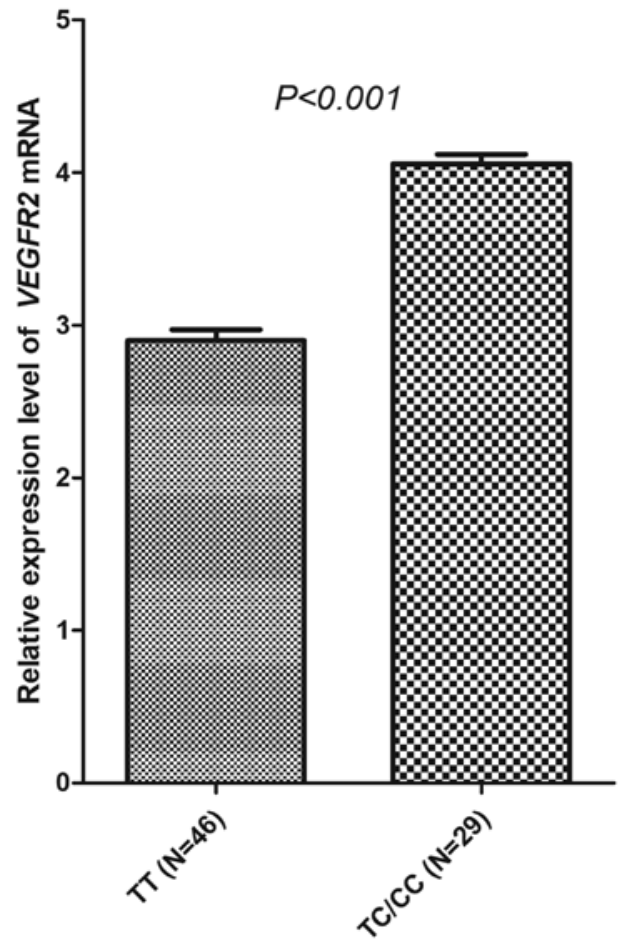

Figure 6. Relative mRNA expression levels of $V E G F R 2$ in 75 peripheral blood mononuclear cells specimens according to VEGFR2 rs2071559 genotype status. VEGFR2, vascular endothelial growth factor receptor 2.

clinical trials (34). Furthermore, patients with an ECOG score of 2 were included in the present study, in contrast to the phase II clinical trial, where these patients were excluded. However, the clinical significance of ECOG score has been confirmed in previous studies, suggesting that the higher the score, the worse the prognosis $(35,36)$. In the present study, the results of Cox's regression analysis indicated that patients with a score of 1-2 were associated with a worse prognosis. Notably, the present median OS time was 15.10 months, which was slightly longer compared with that reported in the phase II clinical trial with a median OS time of 14.50 months (21). It is possible that the reason for this difference may be the licensing of the PARP inhibitor olaparib in 2018. Patients with EOC in China had the opportunity to be treated with olaparib in the subsequent lines of treatment, which was effective and offered an extended survival benefit for the patients (37).
Although apatinib was licensed in China $\sim 5$ years ago, only a few studies have investigated potential predictive biomarkers for the treatment with apatinib. Results from a previous study suggested that the occurrence of proteinuria or hand-foot syndrome during treatment with apatinib could predict superior clinical outcomes $(38,39)$. However, polymorphism studies that could indicate a potential benefit for the use of apatinib are still limited Regarding EOC in particular, one study investigated the effect of polymorphisms in the susceptibility of EOC (40). Previous studies have also indicated that germline polymorphisms may play a role in the development of ovarian cancer $(41,42)$. To the best of our knowledge, the present study was the first to research the effect of the presence of a polymorphism in Chinese patients with EOC receiving apatinib treatment. The result of polymorphism analysis was partly consistent with that of a previous study by Scartozzi et al (43). In the aforementioned study, a total of 148 patients with advanced hepatocellular cancer treated with sorafenib were enrolled and analyzed. Although, the prevalence of the rs 2071559 polymorphism was greater compared with that in the present study ( 0.49 vs. 0.22), the clinical outcomes showed that the prognosis of patients with the TC/CC genotype were worse, which was consistent with the findings of the present study. Additionally, the results of a recent study by Sullivan et al (44) were also in line with those of the present study. In the aforementioned study, patients with advanced non-small cell lung cancer (NSCLC) treated with platinum-based chemotherapy were enrolled, and the polymorphisms of VEGF-A,VEGFRI and $V E G F R 2$ were investigated. The results of multivariate analysis suggested that VEGF-A rs2010963 and VEGFR2 rs2071559 were significantly associated with prognosis. Specifically, patients with the TT genotype of rs2071559 were associated with an improved prognosis, which was consistent with the results of the present study, where the TT genotype was associated with improved PFS and OS times. A recent phase III clinical trial that investigated the effect of adjuvant sunitinib in patients with high-risk renal cell carcinoma (45) suggested that the median disease-free survival time was longer in patients with the TT genotype, which is consistent with the effects observed for the TT genotype in the present study. In conclusion, the VEGFR2 rs2071559 polymorphism might be of clinical significance as a biomarker for the prediction of patients with EOC who 
might show an improved response towards apatinib treatment.

With regard to the safety analysis, results from a previous study indicated that hypertension is usually the most common adverse reaction in patients with cancer receiving apatinib treatment (46). However, the present study reported that hand-foot syndrome was the most common adverse reaction, with an incidence $>40 \%$, which was consistent with the results observed in patients with EOC who received sorafenib therapy (47). It hypothesized that this effect might be attributed to a difference in sex. A previous study suggested that the incidence of hand-foot syndrome and other overall toxicity in women was higher than that in men (48). Additionally, the other adverse reactions classified as grade $\geq 2$ with an incidence $\geq 10 \%$ included hypertension, nausea/vomiting, proteinuria, fatigue and diarrhea, which were similar to the adverse reactions observed in another previous study for apatinib treatment (49). In the present study, the association analysis between genotype status and adverse reactions failed to show any significant difference, which suggested that the polymorphism was not associated with apatinib disposition for specific adverse reactions.

Notably, mRNA expression analysis indicated that the VEGFR2 mRNA expression levels were significantly different according to rs2071559 polymorphism status. VEGFR2 is the receptor with the strongest binding affinity to VEGF-A and has an important role in signal transmission. In addition, the expression levels of VEGFR2 show a crucial role in the process of angiogenesis (50). Moreover, previous studies also suggested that higher expression levels of VEGFR2 in tumor cells were associated with a higher likelihood for tumor cells to relapse and metastasize in gastric cancer and epithelial cancer $(51,52)$. In addition, higher expression levels of VEGFR2 have been associated with worse PFS and OS times in patients with NSCLC (53) and cervical cancer (54), which is consistent with the preliminary results of the present study.

There are a number of limitations to the present study. Firstly, the sample size was small. Secondly, the study was designed as a retrospective analysis, so some bias (no control group and randomization) might be unavoidable. Thirdly, the present study did not perform cell- and animal-based experiments. Lastly, the results of the present study need to be validated in clinical trials. However, the prognostic value of the VEGFR2 rs2071559 polymorphism was fully evaluated, and the determination of VEGFR2 mRNA expression levels might indicate the polymorphism and prognosis of patients with EOC. Therefore, the present study was of clinical significance for the evaluation of prognosis in patients with EOC who received apatinib treatment.

\section{Acknowledgements}

The authors would like to thank Professor Todd Knepper from the America Moffitt Cancer Center for editing and polishing the English language of the original manuscript.

\section{Funding}

No funding was received.

\section{Availability of data and materials}

The datasets used and/or analyzed during the current study are available from the corresponding author on reasonable request.

\section{Authors' contributions}

ZY, YYG, WD and YG conceived and designed the study. ZY, $\mathrm{XDH}, \mathrm{QZ}$ and MW collected the characteristics of the patients and performed the experiments. ZY and HLK analyzed the data. YYG, MW and YG contributed with reagents and analysis tools. ZY, YYG, WD and YG wrote the paper. All authors read and approved the final manuscript and agreed to be accountable for all aspects of the research in ensuring that the accuracy or integrity of any part of the work was appropriately investigated and resolved.

\section{Ethics approval and consent to participate}

This study was approved by the Ethics Committee of Beijing Obstetrics and Gynecology Hospital, Capital Medical University (Beijing, China) (approval no. 2017-KY-01105). Written informed consent was obtained from all patients or their family members. Only in circumstances where the patients had passed away or the patient was unaware of their diagnosis of EOC, was written informed consent obtained from their relatives.

\section{Patient consent for publication}

Not applicable.

\section{Competing interests}

The authors declare that they have no competing interests.

\section{References}

1. Bray F, Ferlay J, Soerjomataram I, Siegel RL, Torre LA and Jemal A: Global cancer statistics 2018: GLOBOCAN estimates of incidence and mortality worldwide for 36 cancers in 185 countries. CA Cancer J Clin 68: 394-424, 2018.

2. Chen W, Zheng R, Baade PD, Zhang S, Zeng H, Bray F, Jemal A, $\mathrm{Yu}$ XQ and He J: Cancer statistics in China, 2015. CA Cancer J Clin 66: 115-132, 2016.

3. Li X, Zhang Y, Chai X, Zhou S, Zhang H, He J, Zhou R, Cai L, Chen L and Tao G: Overexpression of MEF2D contributes to oncogenic malignancy and chemotherapeutic resistance in ovarian carcinoma. Am J Cancer Res 9: 887-905, 2019.

4. Mari R, Mamessier E, Lambaudie E, Provansal M, Birnbaum D, Bertucci $F$ and Sabatier R: Liquid biopsies for ovarian carcinoma: How blood tests may improve the clinical management of a deadly disease. Cancers (Basel) 11: 774, 2019.

5. Liu CL, Pan HW, Torng PL, Fan MH and Mao TL: SRPX and HMCN1 regulate cancer-associated fibroblasts to promote the invasiveness of ovarian carcinoma. Oncol Rep 42: 2706-2715, 2019.

6. Morgan RJ Jr, Armstrong DK, Alvarez RD, Bakkum-Gamez JN, Behbakht K, Chen LM, Copeland L, Crispens MA, DeRosa M, Dorigo O, et al: Ovarian cancer, version 1.2016, NCCN clinical practice guidelines in oncology. J Natl Compr Canc Netw 14: 1134-1163, 2016.

7. Ledermann JA, Raja FA, Fotopoulou C, Gonzalez-Martin A, Colombo $\mathrm{N}$ and Sessa C; ESMO Guidelines Working Group: Newly diagnosed and relapsed epithelial ovarian carcinoma: ESMO clinical practice guidelines for diagnosis, treatment and follow-up. Ann Oncol 29 (Suppl 4): iv259, 2018. 
8. Canaz E, Grabowski JP, Richter R, Braicu EI, Chekerov R and Sehouli J: Survival and prognostic factors in patients with recurrent low-grade epithelial ovarian cancer: An analysis of five prospective phase II/III trials of NOGGO metadata base. Gynecol Oncol 154: 539-546, 2019.

9. Champer M, Huang Y,Hou JY, Tergas AI, Burke WM, Hillyer GC, Ananth CV, Neugut AI, Hershman DL and Wright JD: Adherence to treatment recommendations and outcomes for women with ovarian cancer at first recurrence. Gynecol Oncol 148: 19-27, 2018.

10. Corrado G, Salutari V, Palluzzi E, Distefano MG, Scambia G and Ferrandina G: Optimizing treatment in recurrent epithelial ovarian cancer. Expert Rev Anticancer Ther 17: 1147-1158, 2017.

11. Pujade-Lauraine E, Hilpert F, Weber B, Reuss A, Poveda A, Kristensen G, Sorio R, Vergote I, Witteveen P, Bamias A, et al: Bevacizumab combined with chemotherapy for platinum-resistant recurrent ovarian cancer: The AURELIA open-label randomized phase III trial. J Clin Oncol 32: 1302-1308, 2014.

12. Monk BJ, Randall LM and Grisham RN: The evolving landscape of chemotherapy in newly diagnosed advanced epithelial ovarian cancer. Am Soc Clin Oncol Educ Book 39: e141-e151, 2019.

13. Lin B, Song X, Yang D, Bai D, Yao Y and Lu N: Anlotinib inhibits angiogenesis via suppressing the activation of VEGFR2, PDGFR $\beta$ and FGFR1. Gene 654: 77-86, 2018.

14. Liu J, Nicum S, Reichardt P, Croitoru K, Illek B, Schmidinger M, Rogers C, Whalen C and Jayson GC: Assessment and management of diarrhea following VEGF receptor TKI treatment in patients with ovarian cancer. Gynecol Oncol 150: 173-179, 2018.

15. Burger RA, Sill MW, Monk BJ, Greer BE and Sorosky JI: Phase II trial of bevacizumab in persistent or recurrent epithelial ovarian cancer or primary peritoneal cancer: A gynecologic oncology group study. J Clin Oncol 25: 5165-5171, 2007.

16. Friedlander M, Hancock KC, Rischin D, Messing MJ, Stringer CA, Matthys GM, Ma B, Hodge JP and Lager JJ: A Phase II, open-label study evaluating pazopanib in patients with recurrent ovarian cancer. Gynecol Oncol 119: 32-37, 2010.

17. Chekerov R, Hilpert F, Mahner S, El-Balat A, Harter P, De Gregorio N, Fridrich C, Markmann S, Potenberg J, Lorenz R, et al: Sorafenib plus topotecan versus placebo plus topotecan for platinum-resistant ovarian cancer (TRIAS): A multicentre, randomised, double-blind, placebo-controlled, phase 2 trial. Lancet Oncol 19: 1247-1258, 2018.

18. Li J, Qin S, Xu J, Xiong J, Wu C, Bai Y, Liu W, Tong J, Liu Y, $\mathrm{Xu} \mathrm{R}$, et al: Randomized, double-blind, placebo-controlled phase III trial of apatinib in patients with chemotherapy-refractory advanced or metastatic adenocarcinoma of the stomach or gastroesophageal junction. J Clin Oncol 34: 1448-1454, 2016.

19. Zhao D, Hou H and Zhang X: Progress in the treatment of solid tumors with apatinib: A systematic review. Onco Targets Ther 11: 4137-4147, 2018.

20. Matei D, Sill MW, Lankes HA, DeGeest K, Bristow RE, Mutch D, Yamada SD, Cohn D, Calvert V, Farley J, et al: Activity of sorafenib in recurrent ovarian cancer and primary peritoneal carcinomatosis: A gynecologic oncology group trial. J Clin Oncol 29: 69-75, 2011

21. Miao M, Deng G, Luo S, Zhou J, Chen L, Yang J, He J, Li J, Yao J, Tan S and Tang J: A phase II study of apatinib in patients with recurrent epithelial ovarian cancer. Gynecol Oncol 148: 286-290, 2018

22. Liu S, Wu M, Zhang B, Xiong X, Wang H and Zhou X: Analysis of genetic polymorphisms for age-related macular degeneration (AMD) in Chinese Tujia ethnic minority group. BMC Med Genet 20: 25, 2019.

23. Song ZZ, Zhao LF, Zuo J, Fan ZS, Wang L and Wang YD Clinical outcomes and safety of apatinib mesylate in the treatment of advanced non-squamous non-small cell lung cancer in patients who progressed after standard therapy and analysis of the KDR gene polymorphism. Onco Targets Ther 13: 603-613, 2020.

24. Babyshkina N, Zavyalova M, Tarabanovskaya N, Dronova T, Krakhmal N, Slonimskaya E, Kzhyshkowska J, Choynzonov E and Cherdyntseva N: Predictive value of vascular endothelial growth factor receptor type 2 in triple-negative breast cancer patients treated with neoadjuvant chemotherapy. Mol Cell Biochem 444: 197-206, 2018.

25. Escudier B, Rini BI, Motzer RJ, Tarazi J, Kim S, Huang X, Rosbrook B, English PA, Loomis AK and Williams JA: Genotype correlations with blood pressure and efficacy from a randomized phase III trial of second-line axitinib versus sorafenib in metastatic renal cell carcinoma. Clin Genitourin Cancer 13: 328-337.e3, 2015.
26. Mert I, Kumar A, Torres D, Huang Y, McGree ME, Weaver AL and Cliby WA: Should mucosal bowel invasion in ovarian cancer be assigned to FIGO stage IV disease? Gynecol Oncol 153: 238-241, 2019.

27. Eisenhauer EA, Therasse P, Bogaerts J, Schwartz LH, Sargent D, Ford R, Dancey J, Arbuck S, Gwyther S, Mooney M, et al: New response evaluation criteria in solid tumours: Revised RECIST guideline (version 1.1). Eur J Cancer 45: 228-247, 2009.

28. Miller TP, Fisher BT, Getz KD, Sack L, Razzaghi H, Seif AE, Bagatell R, Adamson PC and Aplenc R: Unintended consequences of evolution of the common terminology criteria for adverse events. Pediatr Blood Cancer 66: e27747, 2019.

29. Livak KJ and Schmittgen TD: Analysis of relative gene expression data using real-time quantitative PCR and the 2(-Delta Delta $\mathrm{C}(\mathrm{T}))$ method. Methods 25: 402-408, 2001

30. Tian Z, Gu Z, Wang X, Liu Z, Yao W, Wang J, Zhang P, Cai Q and $\mathrm{Ge} \mathrm{H}$ : Efficacy and safety of apatinib in treatment of osteosarcoma after failed standard multimodal therapy: An observational study. Medicine (Baltimore) 98: e15650, 2019.

31. Chen X, Qiu T, Zhu Y, Sun J, Li P, Wang B, Lin P, Cai X, Han X, Zhao F, et al: A single-arm, phase II study of apatinib in refractory metastatic colorectal cancer. Oncologist 24: 883-e407, 2019.

32. Liao Z, Li F, Zhang C, Zhu L, Shi Y, Zhao G, Bai X, Hassan S, Liu X, Li T, et al: Phase II trial of VEGFR2 inhibitor apatinib for metastatic sarcoma: Focus on efficacy and safety. Exp Mol Med 51: 1-11, 2019.

33. Qiu H, Li J, Liu Q, Tang M and Wang Y: Apatinib, a novel tyrosine kinase inhibitor, suppresses tumor growth in cervical cancer and synergizes with paclitaxel. Cell Cycle 17: 1235-1244, 2018.

34. Yilmaz E, Sahin N, Koleli I, Melekoglu R, Tanrikut E, Faydali S, Karaer A and Coskun EI: Retrospective analysis of borderline ovarian tumors: Outcomes at a single center. Acta Clin Croat 58 29-36, 2019

35. Agemi Y, Shimokawa T, Sasaki J, Miyazaki K, Misumi Y, Sato A, Aida S, Ishii M, Nakamura Y, Naoki K and Okamoto H: Prospective evaluation of the G8 screening tool for prognostication of survival in elderly patients with lung cancer: A single-institution study. PLoS One 14: e0210499, 2019.

36. Liu JY, Zhu BR, Wang YD and Sun X: The efficacy and safety of Apatinib mesylate in the treatment of metastatic osteosarcoma patients who progressed after standard therapy and the VEGFR2 gene polymorphism analysis. Int J Clin Oncol 25: 1195-1205, 2020.

37. Boussios S, Karihtala P, Moschetta M, Karathanasi A, Sadauskaite A, Rassy E and Pavlidis N: Combined strategies with poly (ADP-Ribose) polymerase (PARP) inhibitors for the treatment of ovarian cancer: A literature review. Diagnostics (Basel) 9: 87, 2019.

38. Liu X, Qin S, Wang Z, Xu J, Xiong J, Bai Y, Wang Z, Yang Y, Sun G, Wang L, et al: Early presence of anti-angiogenesis-related adverse events as a potential biomarker of antitumor efficacy in metastatic gastric cancer patients treated with apatinib: A cohort study. J Hematol Oncol 10: 153, 2017.

39. Fang SC, Huang W, Zhang YM, Zhang HT and Xie WP. Hypertension as a predictive biomarker in patients with advanced non-small-cell lung cancer treated with apatinib. Onco Targets Ther 12: 985-992, 2019.

40. Lawrenson K, Song F, Hazelett DJ, Kar SP, Tyrer J, Phelan CM, Corona RI, Rodríguez-Malavé NI, Seo JH, Adler E, et al: Genome-wide association studies identify susceptibility loci for epithelial ovarian cancer in east Asian women. Gynecol Oncol 153: 343-355, 2019.

41. Narod SA: Personalised medicine and population health: Breast and ovarian cancer. Hum Genet 137: 769-778, 2018.

42. Jin Y: Association between EPHX1 polymorphism rs1051740 and the risk of ovarian cancer: A meta-analysis. Artif Cells Nanomed Biotechnol 47: 2338-2342, 2019.

43. Scartozzi M, Faloppi L, Svegliati Baroni G, Loretelli C, Piscaglia F, Iavarone M, Toniutto P, Fava G, De Minicis S, Mandolesi A, et al: VEGF and VEGFR genotyping in the prediction of clinical outcome for HCC patients receiving sorafenib: The ALICE-1 study. Int J Cancer 135: 1247-1256, 2014.

44. Sullivan I, Riera P, Andrés M, Altés A, Majem M, Blanco R, Capdevila L, Barba A, Barnadas A and Salazar J: Prognostic effect of VEGF gene variants in metastatic non-small-cell lung cancer patients. Angiogenesis 22: 433-440, 2019.

45. George DJ, Martini JF, Staehler M, Motzer RJ, Magheli A, Donskov F, Escudier B, Li S, Casey M, Valota O, et al: Phase III trial of adjuvant sunitinib in patients with high-risk renal cell carcinoma: Exploratory pharmacogenomic analysis. Clin Cancer Res 25: 1165-1173, 2019. 
46. Zhu Y, Feng B, Mei L, Sun R, Guo C and Zhu J: Clinical efficacy of TACE combined with apatinib in the treatment of advanced hepatocellular carcinoma. J BUON 24: 608-614, 2019.

47. Bodnar L, Górnas M and Szczylik C: Sorafenib as a third line therapy in patients with epithelial ovarian cancer or primary peritoneal cancer: A phase II study. Gynecol Oncol 123: 33-36, 2011.

48. Mueller F, Büchel B, Köberle D, Schürch S, Pfister B Krähenbühl S, Froehlich TK, Largiader CR and Joerger M: Gender-specific elimination of continuous-infusional 5-fluorouracil in patients with gastrointestinal malignancies: Results from a prospective population pharmacokinetic study. Cancer Chemother Pharmacol 71: 361-370, 2013.

49. Lan CY, Wang Y, Xiong Y, Li JD, Shen JX, Li YF, Zheng M, Zhang YN, Feng YL, Liu Q, et al: Apatinib combined with oral etoposide in patients with platinum-resistant or platinum-refractory ovarian cancer (AEROC): A phase 2, single-arm, prospective study. Lancet Oncol 19: 1239-1246, 2018.
50. Cho HD, Moon KD,Park KH,Lee YS and Seo KI: Effects of auriculasin on vascular endothelial growth factor (VEGF)-induced angiogenesis via regulation of VEGF receptor 2 signaling pathways in vitro and in vivo. Food Chem Toxicol 121: 612-621, 2018. 51. Lu Y, Xu Q, Zuo Y, Liu L, Liu S, Chen L, Wang K, Lei Y, Zhao X and $\mathrm{Li}$ Y: Isoprenaline/ $\beta 2-\mathrm{AR}$ activates Plexin-A1/VEGFR2 signals via VEGF secretion in gastric cancer cells to promote tumor angiogenesis. BMC Cancer 17: 875, 2017.

52. Jinesh GG, Manyam GC, Mmeje CO, Baggerly KA and Kamat AM: Surface PD-L1, E-cadherin, CD24, and VEGFR2 as markers of epithelial cancer stem cells associated with rapid tumorigenesis. Sci Rep 7: 9602, 2017.

53. Ding M, Liu L, Hu C, Liu Y, Qiao Y and Jiang X: Expression of VEGFR2 and NRP-1 in non-small cell lung cancer and their clinical significance. Chin J Cancer Res 26: 669-677, 2014.

54. Dang YZ, Zhang Y, Li JP, Hu J, Li WW, Li P, Wei LC and Shi M: High VEGFR1/2 expression levels are predictors of poor survival in patients with cervical cancer. Medicine (Baltimore) 96: e5772, 2017. 